\title{
Effect of tart cherry juice on arterial stiffness, inflammation and other risk markers for cardiovascular disease in healthy adults
}

\author{
A. Lynn ${ }^{1}$, S. Mathew ${ }^{2}$, C. T. Moore, J. Russell ${ }^{3}$, E. Robinson ${ }^{2}$, V. Soumpasi ${ }^{2}$ and M. E. Barker ${ }^{2}$ \\ ${ }^{1}$ Food and Nutrition Group, Sheffield Business School, Sheffield Hallam University S1 1WB, ${ }^{2}$ Human Nutrition Unit, \\ School of Medicine, University of Sheffield, S10 2RX \\ ${ }^{3}$ CICS, University of Sheffield S10 $2 \mathrm{HB}, \mathrm{UK}$
}

Inflammation is thought to play an important role in the development of arterial stiffness ${ }^{(1)}$. Evidence indicates that cherries may have anti-inflammatory effects ${ }^{(2,3)}$. Consumption of sweet cherries over a period of $28 \mathrm{~d}$ reduced markers of inflammation in healthy middle aged adults and consumption of tart cherry juice reduced exercise-induced inflammation in marathon runners ${ }^{(2,3)}$. The effect of cherries on arterial stiffness has not been examined.

We conducted an open-label randomised placebo controlled study to determine whether a tart cherry juice concentrate (Cherry Active ${ }^{\circledR}$ ) reduced arterial stiffness, inflammation and risk markers for cardiovascular disease in 46 healthy adults (30-50y). Participants consumed $30 \mathrm{ml}$ of cherry concentrate diluted to a volume of $250 \mathrm{ml}$ with water or the same volume of an energy matched control drink daily for 6 weeks. Measurements were taken at baseline and at the end of the intervention. The primary outcome variable was change in pulse wave velocity (PWV). The secondary outcome variable was change in serum C-reactive protein (CRP; a marker of inflammation). The study was approved by the Ethics Committee of Sheffield Hallam University and all participants provided written informed consent.

Arterial stiffness was measured as brachial-knee pulse wave velocity (Nicolet Vasoguard Microlight system, VIASYS Healthcare, USA). Brachial blood pressure was measured in triplicate using a semi-automated sphygmomanometer (Accutorr PlusTM Datascope, USA). Fasted blood samples were collected at the start and end of the intervention to measure CRP and lipid variables. Serum CRP was measured using a high sensitivity commercial ELISA kit from MP Biomedicals. Total cholesterol, high density lipoprotein cholesterol (HDL) and triacylglycerol (TAG) were measured on a Reflotron Plus reflectance photometer. Low density lipoprotein cholesterol (LDL) was calculated using the Friedewald equation ${ }^{(4)}$. The table shows mean baseline and post intervention values by treatment group. There was no significant effect of the intervention on any outcome measure.

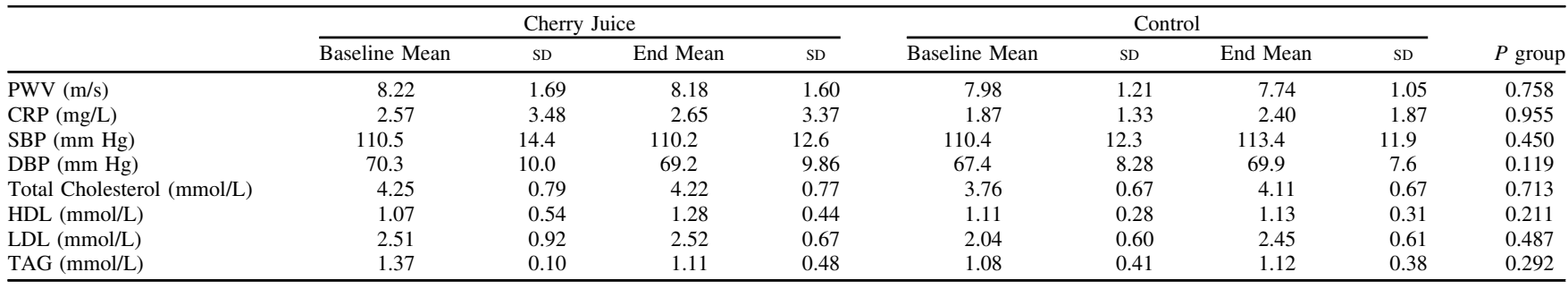

Effect of treatment was assessed by ANCOVA, with baseline and age as covariates. HDL and hsCRP data were log transformed prior to analysis.

We conclude that a tart cherry juice concentrate rich in polyphenols has no effect on PWV, CRP and risk markers for cardiovascular disease in healthy adults.

1. Mäki-Petäjä KM \& Wilkinson IB (2009) Curr Pharm Des 15, 290-303.

2. Kelley DS, Adkins Y, Reddy A et al. (2013) J. Nutr 143, 340-344.

3. Howatson G, McHugh MP, Hill JA et al. (2010) Scand J Med Sci Sports 20, 843-852.

4. Friedewald WT, Levy RI \& Fredrickson DS (1972) Clin Chem 18, 499-502. 